\title{
Lidil
}

Revue de linguistique et de didactique des langues

47 | 2013

Le verbe pour exprimer le temps

\section{Approches du verbe : apports pour la didactique de la grammaire}

Jean-Pierre Sautot et Solveig Lepoire-Duc

\section{(2) OpenEdition}

1 Journals

Édition électronique

URL : http://journals.openedition.org/lidil/3257

DOI : $10.4000 /$ lidil.3257

ISSN : 1960-6052

Éditeur

UGA Éditions/Université Grenoble Alpes

\section{Édition imprimée}

Date de publication : 31 mai 2013

Pagination : 15-19

ISBN : 978-2-84310-247-9

ISSN : $1146-6480$

\section{Référence électronique}

Jean-Pierre Sautot et Solveig Lepoire-Duc, « Approches du verbe : apports pour la didactique de la grammaire », Lidil [En ligne], 47 | 2013, mis en ligne le 01 décembre 2014, consulté le 23 septembre 2020. URL : http://journals.openedition.org/lidil/3257 ; DOI : https://doi.org/10.4000/lidil.3257

(c) Lidil 


\title{
Approches du verbe : apports pour la didactique de la grammaire
}

\author{
Jean-Pierre Sautot* et Solveig Lepoire-Duc**
}

La notion de verbe est complexe et résiste aux simplifications que les pratiques scolaires de la grammaire tentent de lui appliquer. Cette livraison de la revue a pour objectif de revisiter le concept de verbe dans la perspective de sa transposition didactique, et se propose de rendre compte des descriptions linguistiques existantes pour contribuer à une approche didactique renouvelée. La publication se situe dans la lignée d'autres études comme Le verbe dans tous ses états (Leeman, 2004), la revue Synergies France $\left(\mathrm{n}^{\circ} 6,2010\right)$ : «Verbe, grammaire et enseignement : la prescription et l'usage », ou les journées d'étude Grammatica : «Le verbe : perspectives linguistiques et didactiques» (Avezard-Roger \& Lavieu, à paraitre).

Pour autant, le présent fascicule ne suffira pas à redéfinir le verbe. En revanche, il contribue à redessiner les contours d'une didactique de la grammaire en s'appuyant sur le problème que pose aux praticiens la résolution en classe de la complexité conceptuelle du verbe. Si l'on tente de construire le champ conceptuel du verbe, indépendamment de la langue visée, on obtient un copieux catalogue de catégories que le verbe peut porter : temps, mode, aspect, évidentialité, négation, modalité, articulation, discursive, voix, nombre, registre... (Creissels, 2006). À l'évidence les potentialités sémantiques du verbe sont vastes.

En l'espèce,

Toute grammaire de référence d'une langue devrait se présenter à la fois comme une somme organisée des connaissances ou des savoirs que les locuteurs ou les spécialistes ont sur cette langue, et comme un recensement des usages effectifs que font les locuteurs de cette langue dans les divers registres selon lesquels elle est utilisée. (Deulofeu \& Debaisieux, 2012, p. 27)

* Université Claude Bernard-Lyon 1.

** IUFM, Laboratoire ICAR-Lyon 2. 
Toute didactique de la langue, et donc de sa grammaire, devrait préconiser une éducation aux usages effectifs de cette langue et une instruction aux outils permettant ces usages. Or il convient que la description précède la préconisation et, puisque la grammaire des usages contemporains de la langue reste à écrire, du moins à parfaire, la didactique de la grammaire reste en grande partie à construire dans ce domaine.

Les programmes scolaires tendent souvent à réduire la situation temporelle à la chronologie et à assimiler le temps linguistique à la conjugaison. Or, le «temps» est une catégorie conceptuelle qu'il est nécessaire de traiter en tant que telle, dans ses rapports plurivoques avec les différentes catégories formelles qui servent à l'exprimer (Charaudeau, 1992). On ne s'intéresse ici qu'à l'une de ces catégories : le verbe et sa mise en fonctionnement dans l'expression du temps. Faisant cela, on risque de verser dans une linguistique appliquée qui ne prendrait pas en compte les apports de la psycholinguistique cognitive ou génétique, les apports des sciences de l'éducation, de la didactique du français elle-même. Les contributions présentées ici n'évitent pas complètement l'obstacle, mais, si l'on sait aujourd'hui que les acquisitions langagières jouent un rôle dans le développement du temps conventionnel et dans l'élaboration des cadres aspectuels et temporels (Tartas, 2009), l'impact des enseignements de et sur la langue reste assez méconnu. Ce chantier reste donc ouvert.

À l'école, l'approche morphosyntaxique perdure cependant que les sciences du langage progressent. Ainsi, l'écart entre une description scientifique et une description scolaire du verbe s'accroit. Le besoin d'une approche rénovée de la notion de verbe est patente. Ce besoin est d'autant plus criant que la pédagogie de la grammaire reste fortement ancrée dans une tradition qui date d'avant l'émergence des sciences du langage dans le domaine scientifique. Ce numéro prend le parti d'une approche centrée sur le statut du verbe dans la construction et l'expression du temps, envisagé du point de vue de la chronologie et de l'aspect. Le temps n'est alors qu'une porte d'entrée possible dans la problématique didactique. Partant du verbe et des catégories qu'il porte, est-il possible de dessiner les contours d'une didactique qui rapproche la grammaire scolaire d'une grammaire des usages réels, et qui prenne un peu de distance vis-à-vis des considérations morphosyntaxiques?

Si l'on s'arrête un temps, à titre illustratif, sur l'école française, la carte conceptuelle du verbe apparait comme composée de fragments de théories plus ou moins disparates. Force est d'y constater une dispersion mais surtout des lacunes. S'agissant de la situation des procès 
dans le temps, l'approche chronologique y est majoritaire, voire exclusive. Si on y adjoint l'entrée morphologique, la catégorie du temps se réduit fort vite à la notion de conjugaison. Or la notion de temps et les catégories grammaticales qui lui correspondent appellent plus d'outils - et par suite de concepts grammaticaux - que l'idée de conjugaison n'en suggère. Il convient donc de commencer ici à lister ces concepts, à envisager la manière dont les élèves les appréhendent, à décrire les problèmes que soulèvent ces concepts et ces conceptions pour une transposition dans la classe.

La notion d'infinitif, premier exemple, abordée à l'école française comme une forme atemporelle du verbe, voire comme sa forme lexicalisée, est pourtant considérée par Benveniste (1974) comme la forme modalisée du verbe, au moyen d'un auxiliaire, ou comme une virtualité de réalisation du procès par Guillaume (1929). Quid des formes non fléchies du verbe, parmi lesquelles l'infinitif ? C'est la question à laquelle s'attachent F. Torterat et C. Péret.

F. Torterat s'intéresse à l'apport des formes non fléchies dans l'expression du temps. Faisant cela, il extrait la question de l'expression de la temporalité par le verbe de la seule entrée morphosyntaxique, ce qui revient à questionner la «didactique de la conjugaison». Quant aux initiatives didactiques des auteurs de manuels dans le domaine, la conclusion de l'article montre combien les réticences sont fortes à l'abandon d'une pédagogie du verbe construite sur la conjugaison.

C. Péret aborde la question de l'auxiliarisation du verbe et plus particulièrement dans l'expression du futur. Elle étudie les grammaires scolaires et montre comment l'école française exclut progressivement de son champ de travail une forme très usitée, telle le futur périphrastique, niant au passage le statut d'auxiliaire au verbe «aller».

Ainsi la notion de verbe auxiliaire est-elle le plus souvent ramenée à la simple construction de temps verbaux dits composés, négligeant totalement les autres acceptions du concept d'auxiliaire telles que proposées par Benveniste (1974) ou Creissels (2006). C. Corteel, C. AvezardRoger et C. Lachet se confrontent, entre autres, aux constructions complexes du verbe qui usent d'auxiliaires bien au-delà de la définition restreinte aux verbes «être» et «avoir» et esquissent des pistes de transposition didactique.

Dans l'expression du temps, les outils linguistiques rendant compte de la réalisation du procès qu'exprime le verbe sont fréquemment oubliés. Quid du concept d'aspect, qu'explorent C. Lachet, C. Corteel et C. Avezard-Roger, quand l'expression du temps est fréquemment 
réduite aux divers tiroirs verbaux répartis dans une chronologie passéprésent-futur, là où la Grammaire du sens de Charaudeau (1992) propose une dizaine de « visions » pour rendre compte de la situation dans le temps?

C. Corteel et C. Avezard-Roger questionnent les formes verbales périphrastiques au travers d'interprétations d'élèves de l'école élémentaire. Ces interprétations montrent que les valeurs aspectuelles contenues dans certaines formes périphrastiques, comme «venir de» ou «aller»+ infinitif, sont comprises sinon toujours correctement interprétées. Cette compréhension parfois intuitive montre l'intérêt qu'il y aurait à aborder le concept d'aspect en classe.

C. Lachet analyse les manuels de français destinés à l'enseignement secondaire. Elle questionne l'enseignement possible de l'aspect verbal et trace des propositions pour une transposition des savoirs scientifiques dans la classe. C. Lachet argumente ainsi un travail du verbe dans le texte plutôt que dans la phrase. Les temps du récit, ou du discours, sont d'ailleurs fréquemment pensés comme des usages phrastiques étendus au texte. À ce sujet, Rastier (2008) distingue clairement deux types de règles d'usages. S'appuyant sur Saussure, F. Rastier réserve le terme de norme aux productions textuelles ou discursives et cantonne la règle à un niveau morphosyntaxique. De manière plus subtile cependant, ce raisonnement indique que des règles constitutives agiraient dans des systèmes tels la syntaxe, la morphologie, quand des normes agiraient sur les relations entre ces systèmes constitutifs. Deux niveaux normatifs sont à l'œuvre lors de la production textuelle qu'il conviendrait de ne pas confondre sur le plan didactique.

Dans une même perspective, R. Gagnon et C. Rodríguez Gonzalo, respectivement à propos du texte argumentatif en français et du texte narratif en espagnol, s'intéressent aux normes qui régissent les usages du verbe. Les deux auteures questionnent la compétence des élèves sur les deux plans normatifs pour proposer ou défendre des dispositifs didactiques.

\section{RÉFÉRENCES BIBLIOGRAPHIQUES}

Avezard-Roger, C. \& Lavieu, B. (dir.). (À paraitre). Le verbe : perspectives linguistiques et didactiques. Arras : Artois Presses Université.

Benveniste, É. (1974). Problèmes de linguistique générale. Paris : Gallimard. 
Charaudeau, P. (1992). Grammaire du sens et de l'expression. Paris : Hachette.

Chervel, A. (2006). Histoire de l'enseignement du français du XVII ${ }^{e}$ au XX siècle. Paris : Retz.

Creissels, D. (2006). Syntaxe générale, une introduction typologique. Paris : Lavoisier.

Deulofeu, H.-J. \& Debaisieux, J.-M. (2012). Une tâche à accomplir pour la linguistique française du $\mathrm{xxI}^{\mathrm{e}}$ siècle : élaborer une grammaire des usages du français. Langue française, 176, 27-46.

Guillaume, G. (1929). Temps et Verbe. Théorie des aspects, des modes et des temps. Paris : H. Champion.

Leeman, D. (dir.). (2004). De la langue au texte. Le verbe dans tous ses états. Namur : Presses universitaires de Namur.

Lepoire-Duc, S. \& Ulma, D. (dir.). (2010). Verbe, grammaire et enseignement : la prescription et l'usage. Synergies France, 6, revue du GERFLINT. Repéré à http://ressources-cla.univ-fcomte.fr/gerflint/France6/ france6.html

Rastier, F. (2008). Conditions d'une linguistique des normes. Texto! Repéré à http://www.revue-texto.net/index.php?id=1612

TARTAs, V. (2009). La construction du temps social par l'enfant. Recherches en sciences de l'éducation (Vol. 143). Berne : Peter Lang. 